\title{
Interrupted Time Experience in Autism Spectrum Disorder - Empirical Evidence from Content Analysis
}

\author{
David Vogel1, Christine M. Falter-Wagner2,3,4 ,Theresa Schoofs1, Katharina Krämer1, Christian \\ Kupke5, Kai Vogeley1,6
}

1 Department of Psychiatry, University of Cologne, Kerpener Street 62, 50924 Cologne, Germany

2 Department of Psychiatry, Medical Faculty, LMU Munich, Nußbaumstr. 7, 80336 Munich, Germany

3 Institute of Medical Psychology, Medical Faculty, LMU Munich, Goethestr. 31, 80336 Munich, Germany

4 Department of Psychology, University of Cologne, Gronewaldstraße 2, 50931 Cologne, Germany

5 Department of Psychiatry, Society for Philosophy and Sciences of the Psyche, Charité, Humboldt-University Berlin, Germany

6 Institute for Neuroscience and Medicine, Cognitive Neuroscience (INM-3), Research Centre Juelich, 52425 Juelich, Germany

\begin{abstract}
Although the experience of time is of central relevance for psychopathology, qualitative approaches to study the inner experience of time have been largely neglected in autism research. We present results from qualitative data acquired from 26 adults with high functioning autism spectrum disorder (ASD). Employing inductive content analysis we identified a distinct pattern of interrupted time experience in ASD. Individuals with ASD seemed to implement structured and routine behavior by future planning to guarantee that the present passed uninterrupted. We reason that the success of corresponding compensatory mechanisms determines the development of distress and noticeable symptoms. Considering recent theories on Bayesian perceptual inference we relate the syndrome of interrupted time experience to the putative neuronal mechanisms underlying time experience.
\end{abstract}

Keywords: autism spectrum disorder, time experience, perceptual inference, psychopathology, content analysis

\section{Introduction}

Human beings both act and interact in time. Emphasizing the temporal character of interaction, the experience of time has repeatedly been linked to intersubjectivity (Fuchs, 2001; Wyllie, 2005; Vogeley \& Kupke, 2007; Botbol et al., 2013; Fuchs, 2013; Mölder, 2016; Nielsen, 2017), and psychopathological disturbances of time experience repeatedly have been associated with disturbances of intersubjectivity on a temporal level (Fuchs, 2005; Wyllie, 2005; Fuchs, 2013; Moskalewicz, 2015a; Moskalewicz, 2015b; Stanghellini et al. 2016; Fuchs \& van Duppen, 2017; Nielsen, 2017). These observations and hypotheses suggest an intricate relationship between time experience and social behavior.

Autism spectrum disorder (ASD) is a psychopathological condition exhibiting "deficits in social communication and social interaction across multiple contexts", and "restricted, repetitive patterns of behavior, interests, or activities" (American Psychiatric Association 2013, p.50). Taking a closer look at the two primary symptom clusters of ASD, we observe on the one hand, deficits in social interaction and on the other hand, deficits in what may be classified as temporal behavior and structure either through repetition or insistence on sameness (Allman et al. 2011; Falter et al. 2012a; Gil et al. 2012). Non-surprisingly, ASD has received increasing interest from scientists and researchers interested in disturbances and alterations in time perception, time production and time experience.

Concerning these various aspects of human time, a multitude of results has repeatedly demonstrated atypicalities in ASD: disturbed neuronal timing (Brock et al., 2002; Welsh et al. 2005), impaired perception of time intervals (for review see Allman \& Falter, 2015; Allman \& Mack 2012; Falter \& Noreika 2014), alterations of perceptual temporal resolution and sensory 
integration windows (Bebko et al. 2006; Foss-Feig et al. 2010; Falter et al. 2012b; Gepner \& Féron, 2009; Woynaroski, 2013; Noel et al., 2017), temporal acuity (Noel et al., 2018), abnormal neuronal processing of time experience (e.g., Lepistö et al. 2005, Lepistö et al. 2006; Rippon et al. 2007; Falter et al. 2013), an impairment in so-called diachronic thought (Boucher et al., 2007), anomalies in clock genes and circadian timing (Wimpory et al., 2002; Nicholas et al., 2007), and abnormal temporal synchrony of behavior in interactions (e.g. Trevarthen \& Daniel, 2005). It has previously been argued that these anomalies may relate to the above mentioned primary symptoms of ASD (Boucher J, 2001; Gepner \& Féron, 2009; Allman, 2011; Allman et al., 2011; Tordjman et al., 2015), however, as of yet definitive conclusions cannot be drawn.

It has recently been suggested, that temporal flow and hence time experience may be constituted by the continuous processes composing Bayesian perceptual inference (Hohwy et al. 2016; also see Vogel et al., 2018a). Bayesian inference states that top-down inferences regarding our environment, called priors, are constantly updated by the neural system depending on bottomup perceptual information (e.g. Friston et al., 2014; Petzschner et al., 2017). The active prior reflects what the neural system presently considers important and which hence is registered as conscious experience. The priors are activated according to a hierarchy with lower priors reflecting fine-grained beliefs and higher priors reflecting coarse-grained, general beliefs. An inference is changed as soon as an active prior no longer provides the best explanation for an incoming perceptual input. In this case, a new, better fitting prior is selected. It has been speculated that the transfer from one prior to the next generates temporal flow (Hohwy et al. 2016). Furthermore, it has been speculated, that so-called hypo-priors (Pellicano \& Burr 2012, Hohwy et al. 2016), i.e. priors which carry more perceptual detail and consider important a larger amount of perceptual input, are the underlying cause for symptoms in ASD (Hohwy et al. 2016). Hypo-priors lead to a more permeable perceptual filter and more detailed perceptual information (Hohwy et al. 2016). More detailed perceptual information in individuals with ASD will need more time to be processed as compared to the corresponding, less detailed perceptual input in persons without ASD (Pellicano \& Burr 2012) and the implicit flow of neural information comparatively may lead to a decrease in the velocity of the integration of perceptual input (Hohwy et al. 2016). Consequently the speed at which the external world changes - especially with regards to other persons - will seem faster; the implicit flow of time will speed up (Gepner \& Féron 2009; Hohwy et al. 2016).

Please note, that it is not the consciously experienced velocity of the passage of time that increases. In the case of Bayesian perceptual inference we describe implicit neural processes not accessible to conscious experience. However, these implicit processes are themselves liable to time, as it takes these processes a certain time to be completed (Varela, 1999; Vogeley \& Kupke, 2007). It is these basic processes on a deeper and more fine-grained layer of time which are considered to take longer in ASD due to a higher perceptual density.

These considerations suggest a considerable significance of the implicit processes underlying time experience for ASD. Still, primary symptoms of ASD remain defined within a framework of inner experience and behavior. Yet, the focus of the aforementioned studies was either on individuals' behavior in time or their judgments of temporal aspects of stimuli sparing an important building block of experience and behavior, namely temporality, the very introspective experience of being in time and of time itself.

Hence, the aim of the current study is a comprehensive investigation of time experience in ASD by means of a qualitative research approach. As a mentionable exception to the so far merely quantitative research in the field, Zukauskas et al. (2009) phenomenologically analyzed qualitative data from interviews with 13 participants diagnosed with ASD and found that their participants demonstrated a "factual experience" (p.95) when referring to time: the present was described through specific and isolated facts, being a "prolongation" of the past (p.104); periods of time were described in reference to repetitive behavior and routine; the general concept of time relied on clocks and calendar dates, and it was dominated by the order of activities and the sequence of routines; the future was described in stereotypical terms and was devised of specific facts like isolated routines. The authors reported the "fullest sense of time" (p.103-104) for the experience of 
the past and concluded, that individuals with ASD focus on facts without an extended temporal perspective. Focusing specifically on the sense of past, present, and future, the authors did not include the experience of the passage of time in their investigation. Nevertheless their findings denote a unique experience of time in ASD, related to symptomatology and possibly in accordance with neuropsychological findings and considerations.

The experience of time may pose a deep but distinct phenomenon to ASD. Notwithstanding the probable relationship between time experience, social interaction, and their disturbance in ASD, the link between them has not been investigated and adequate quantitative measures are not yet available. From a clinical and diagnostic standpoint this indicates further psychopathological investigation to foster better understanding of time experience in ASD and to prepare a potential diagnostic resource.

We present qualitative data on the experience of time obtained from participants with ASD. We compare our findings with those from a previous study employing the same methodological approach on healthy participants (Vogel et al., 2018a), in order to potentially identify and discuss distinct differences between individuals without ASD and without history of mental disease. This explorative approach holds the potential to not only provide additional understanding of the experience of adults with ASD, but also to generate novel hypotheses for further quantitative and experimental research.

\section{Material and Methods}

\subsection{The Time Questionnaire}

We employed the Time Questionnaire (TQ) (Figure 1), an open question questionnaire specifically designed for qualitative inquiry into the experience of time. .

- Fig. 1 app. here -

The TQ is based on a conceptual distinction within the conscious experience of time between the flow or passage of time and the structure of time (past, present, future). This distinction is by no means trivial, as it has previously been speculated that differentiating between the flow of time, and the structure of time, may be useful in order to differentiate psychopathological conditions (Kupke, 2007; Kupke \& Vogeley, 2009; Fuchs, 2013; Fuchs \& van Duppen, 2017; Stanghellini et al., 2017; Vogel et al., 2018a). Questions (Q) 1-3 address the experience of the passage of time. Q1 is phrased as open as possible, whereas Q2 further inquires into intersubjective and interactional variance and Q3 addresses situational changes in experience. Q4 through Q7 were designed to address the three temporal dimensions of time (past, present, and future) and related experience and concepts. Additional space was provided to participants to write anything they further deemed

relevant.

Participants were instructed to write as much or as little as they found sufficient to answer each question. No time limits were given in order to guarantee data adequacy through proper data saturation (Sandelowski, M., 1995; Pope, C. et al. 2000; Mason, M., 2010; Glaser B., \& Strauss A., 2017). The TQ has been successfully used in two recent studies (Vogel et al., 2018a; Vogel et al., 2018b) and been demonstrated to sufficiently address both passage and structure of time with differential diagnostic resolution.

\subsection{Participants}

Volunteers were recruited through the Autism Outpatient Clinic at the Department of Psychiatry, University Hospital Cologne, Germany. The Outpatient Clinic provides diagnostic services, selfhelp groups and cognitive-behavioral therapy based group therapy. For research purposes German native speakers between 18 and 65 may volunteer for study participation after confirmed diagnosis 
via an e-mail distribution list. Before study inclusion all volunteers were clinically rescreened in a psychiatric diagnostic interview by the principal investigator qualified for establishing clinical diagnosis $(\mathrm{KV})$. Participants were included if they met diagnostic criteria for Asperger Syndrome (acc. to ICD-10, World Health Organization, 1992), and if they had no record of comorbid neurological or comorbid psychiatric disease, including organic brain disease, mental retardation (IQ<70), bipolar disorder, psychosis, schizophrenia, anxiety disorders, major depressive disorder, and addiction. Participants were included if they were not taking any neuropsychiatric or any otherwise psychoactive or illegal drug over the period of investigation. Although the diagnostic interview was the primary criterion of inclusion we acquired psychometric data from all participants for additional information. Retrospective scores from the AutismSpectrum Quotient (AQ), a 50-item questionnaire designed to assess autistic spectrum traits in the general population (Baron-Cohen et al., 2001) were available for $n=17$ included participants. Although its validity has not been sufficiently established for ASD (Cassidy et al., 2018), we administered the Beck-Depression-Inventory (BDI-II) (Hautzinger et al., 1995), a self-assessment multiple choice test to all participants in order to account for depressive symptoms potentially interfering with time experience (Thönes \& Oberfeld, 2015; Vogel et al., 2018b). We administered a multiple choice vocabulary test for verbal intelligence (WST) (Schmidt \& Metzler, 1992) to all participants in order to guarantee sufficient language proficiency and rule out potentially interfering speech development disorders. Participants were matched to the healthy population reported in Vogel et al. (2018a) according to verbal IQ in order to rule out any confounding influence from group differences in verbal communication. Demographics and results from AQ, BDI-II and WST are presented in Table 1.

- Tbl. 1 app. here -

\subsection{Qualitative Analysis}

All returning documents were analyzed according to summarizing content analysis (SCA; Mayring, 2014) using inductive content analysis (Pope, C et al. 2000). This hypothesis-free approach was considered to be the most adequate due to the lack of sufficient prior investigation into qualitative time experience in adults with ASD. The aim of the explorative methodology was to generate a comprehensive data based description for future quantitative and experimental studies by giving a qualitative description as detailed as possible.

According to SCA, the material was screened for unnecessary examples, redundancies, and repetitions, which were deleted. The remaining text was, if necessary, paraphrased into understandable common language by correcting incorrect orthography or grammar. From the paraphrased material, single statements were identified. For the purpose of coding the whole material, descriptive, categories were formed for each single statement. Statements could range from a single word to a complete answer to a single question of the questionnaire. After all statements of one participant had been assigned to a category, the next participant was coded. After all participants had been coded in this way, the material was re-screened, and categories of similar or identical content were merged. This process was repeated until a reliable categorical system had been established. The material saturated at 26 participants, meaning that further inclusion had not yielded any additional information (Sandelowski, M., 1995; Pope, C. et al. 2000; Mason, M., 2010; Glaser B., \& Strauss A., 2017).

To check the categorical system for reliability, two naïve inter-coders (K.K., T.S.) were provided with the paraphrased material and a descriptive table, consisting of an explication, a coding rule, and an anchor example for each of the established categories (Table 2). Each intercoder then independently coded the material. From the original code and both inter-coder results we 
calculated Krippendorff's alpha (Krippendorff, 2004; Hayes \& Krippendorff, 2007) in order to evaluate inter-coder agreement.

- Tbl. 2 app. here -

\section{Results}

We identified a total of 285 statements which were coded according to 36 categories. The categories were arranged in five groups. The first group referred to the passage of time (101 statements; categories K01-K15). The second group concerned the experience of the present (69 statements; categories K16-K23). The third group addressed the experience of the past (49 statements; categories K24-K30). The fourth group concerned the experience of the future (56 statements; K31-35). The last group was labelled Rest and contained a single category coding for all statements which did not fit any other provided category ( 8 statements; K36). The calculated intercoder agreement of Krippendorff's alpha resulted in a strong agreement of $\alpha=0.86$.

\subsection{Categories within the Group concerning the Passage of Time}

Among the 101 statements within 15 categories addressing the experience of the passage or flow of time, one category (category $\mathrm{K} 15 ; \mathrm{n}=6,23 \%$ of participants) described the experience of time as generally flowing and passing. Several categories (K06-K08, K14; in total 22 statements) referred to general changes or alterations in the passage of time unrelated to situational variance including a generally imprecise sense of time (category K07; $\mathrm{n}=10,38 \%$ of participants) and the necessity of a clock to experience the passage of time (category $\mathrm{K} 08 ; \mathrm{n}=3,12 \%$ of participants). Time was experienced as passing generally fast (category $\mathrm{K} 14 ; \mathrm{n}=4,15 \%$ of participants) or passing with variable velocities, without mentioning any influential factors (category K06; $\mathrm{n}=5,19 \%$ of participants).

Seven categories (categories K01-K04, K09, K13; in total 56 statements) contained statements reporting changes in the experience of time in relation to situational changes: the passage of time sped up during pleasant activities (category $\mathrm{K} 01$; $\mathrm{n}=10,38 \%$ of participants) or during engaging and interesting activities (category K02; $n=9,35 \%$ of participants); the experience of the passage of time appeared to fade or disappear during engaging activities (category K03; $\mathrm{n}=6$, $23 \%$ of participants) or to slow down during unpleasant situations (category K04; $\mathrm{n}=14,54 \%$ of participants). Structured and routine behavior also substantially influenced the passage of time (category K13; $n=12,46 \%$ of participants), resulting either in a pleasant effect on the experience of time $(n=3)$, in a "normalized" experience of time $(n=3)$ or decreasing an otherwise unpleasant experience of time $(n=3)$, stress or surprise $(n=4)$. Structure and routine behavior could also result in changed velocity of passing time, either as slowing down $(n=3)$ or speeding up $(n=1)$. The passage of time was experienced as going faster with age (category K09; n=5, $19 \%$ of participants).

Four categories (category K05, K10-K12; in total 30 statements) referred to changes in the experience of the flow of time during social situations. Although the influence of social encounters on time experience was denied by 7 participants (category $\mathrm{K} 05 ; \mathrm{n}=7,27 \%$ of participants), passage of time was also experienced to change in social contexts, either with decreasing (category K10; $\mathrm{n}=7,27 \%$ of participants) or increasing velocity of the passage of time (category K11; $\mathrm{n}=5,19 \%$ of participants). Four individuals (category $\mathrm{K} 12 ; \mathrm{n}=4,15 \%$ of participants) even stated that during social interaction the passage of time was not registered or felt.

\subsection{Categories within the Group concerning the Present}


At a first superordinate level, categories addressing the present could be assigned to two diverging attitudes. The present was either experienced as a "snapshot" in the sense of a time point (categories $\mathrm{K} 16, \mathrm{~K} 19, \mathrm{~K} 20$; in total 25 statements) or as an extended duration (categories K17, K18, K21, K23; in total 36 statements). The categories relating to snapshot perceptions described the present as a point in time or as lacking duration (category $\mathrm{K} 16$; $\mathrm{n}=8,31 \%$ of participants), as a current, momentary instant (category $\mathrm{K} 19, \mathrm{n}=11,42 \%$ of participants) which cannot be consciously experienced and which is not real (category $\mathrm{K} 20, \mathrm{n}=8,31 \%$ of participants). The seemingly deviating experience of the present as an extended period of time (category K17, $\mathrm{n}=11,42 \%$ of participants), related to the present as being identifiable with a currently performed activity of varying duration (category $\mathrm{K} 18, \mathrm{n}=9,35 \%$ of participants) which meant refuge and sanctuary from external events (category $\mathrm{K} 21, \mathrm{n}=4,15 \%$ of participants) and had to be utilized and spent in a meaningful manner (category K23; $\mathrm{n}=10,38 \%$ of participants).

On a different note, two participants (category K22; $\mathrm{n}=2,8 \%$ of participants) deemed time as generally unimportant. One of these participants experienced time in general as unimportant, and the other referred to the past, present, and future as meaningless.

\subsection{Categories within the Group concerning the Past}

Almost all of the categories concerning the past referred to it as being related to memories and memory content (categories $\mathrm{K} 24, \mathrm{~K} 25, \mathrm{~K} 27-\mathrm{K} 30$; in total 44 statements) if not proposing identity between past and memory (category $\mathrm{K} 24 ; \mathrm{n}=8,31 \%$ of participants). Memory was experienced as good, vivid or specific memory (category K30; $n=6,23 \%$ of participants). Memories of the past had shaped and influenced or had had an effect on their personality (category K27; $\mathrm{n}=12,46 \%$ of participants), the valence of which varied ranging from primarily negative experiences of past recollections (category $\mathrm{K} 28 ; \mathrm{n}=4,15 \%$ of participants) to a positive experience of past memories (category K29; $\mathrm{n}=11,42 \%$ of participants). Accordingly, it was acknowledged that interpretations of past memories could vary (category $\mathrm{K} 25 ; \mathrm{n}=3,12 \%$ of participants). The only category not directly or literally referring to the past as memories (category K26; $\mathrm{n}=5,19 \%$ of participants) stated that the past was over and could not be changed.

\subsection{Categories within the Group concerning the Future}

The two most common categories were concerned with the future that was experienced as uncertain (category $\mathrm{K} 31 ; \mathrm{n}=14,54 \%$ of participants) and that it had to be minutely planned accordingly (category $\mathrm{K} 32 ; \mathrm{n}=14,54 \%$ of participants). The future was associated with fear, anxiety, and imminence (category K34; $\mathrm{n}=12,46 \%$ of participants), but also with hope and pleasant anticipation (category K33; $\mathrm{n}=10,38 \%$ of participants). Feelings of uncertainty and feelings of fear (categories $\mathrm{K} 30$, K34; in total 26 statements) seemed to covary: uncertainty in category K31 was related to a sense of fear $(n=2)$, whereas expressing fear in category K34 was related to the feeling of uncertainty $(n=3)$. Additionally, within the category concerning feelings of fear (category K34) three participants specified these feeling as being directed toward the near and immanent future. Accordingly, six statements (category $35 ; n=6,23 \%$ of participants) generally divided the future into a near future and a more distant future.

\section{Discussion}

In Accordance with the TQ, we will discuss our analysis with regards to the flow or passage of time and the structure of time (past, present, future) for adults with ASD. 


\subsection{The Experience of the Passage of Time}

The experience of the passage of time in individuals with ASD exhibits several features also associated with the experience of time in a population of individuals without autism (Vogel et al, in press). Just as in healthy individuals, time was experienced to pass or flow, to pass generally quickly, and to pass more quickly with increasing age. The participants with ASD described herein experienced changes in the passage of time depending on the affective imprint of a current situation: the experienced velocity of the passage of time would decrease in unpleasant situations, and increase in pleasant situations, just as healthy individuals reported (Vogel et al, in press). Surprisingly, in our qualitative approach there seemed to be no clear difference concerning the experience of time in individuals with ASD depending on the presence or absence of others or the interaction with others. A considerable number of statements explicitly denied any influence of social aspects on time experience. Due to the lack of a clear distinguishability between the experience of time during social situations and non-social situations through experiential accounts, we speculate, that it is not a social situation in itself which influences the passage of time, but that it is most likely the affective imprint of social situations which causes the passage of time to either speed up or slow down, just as reported for healthy non-autistic individuals (Vogel et al, in press). This does not principally rule out social interaction or intersubjectivity as a potential influential factor on time experience in ASD. Our method of employing a questionnaire is directed at experiences spontaneously reported by our participants. While we consider our data saturated (Sandelowski, M., 1995; Pope, C. et al. 2000; Mason, M., 2010; Glaser B., \& Strauss A., 2017), the TQ cannot directly detect the more implicit aspects of time experience (Vogel et al., 2018a).

The first distinct revelation concerning the experience of the passage of time in ASD is the importance of one's personal interest and the ability to delve into an activity. Interesting activities caused the flow of time to speed up. Some individuals with ASD reported that activities also let the sense of time or the passage of time vanish or disappear. This observation was also reported during social interaction. This experience can be identified with what has been called flow experiences (Csikszentmihályi, 1990). Although these experiences may also be detected in healthy individuals (Vogel et al, in press), flow seemed to be of special importance to our participants with ASD. Due to its comparatively frequent appearance in the material $(n=12)$, it can be speculated that the experience of timelessness associated with flow experience either may be a common byproduct to structure and routine behavior or even a generally more desirable state for individuals with ASD. This notion is further highlighted by reports of an unimportance of time $(n=2)$, a generally bad sense of time $(n=10)$ or the need to use clocks or other temporal aides to experience the passage of time $(n=3)$. Accordingly, the inability of individuals with ASD to tell time, especially without clocks or calendars, repeatedly has been reported within the literature (Boucher, 2001; Boucher et al. 2007; Allman \& deLeon, 2009).

Similarly, and as initially hypothesized, the implementation of structured behavior and routine were reported to be directly related to the passage of time (category K13; n=12). Routine, structured or stereotypical behavior and special interests made the passage of time more pleasant, suppressed the sense of time and prevented negative feelings.

In summary, the passage of time in ASD is experienced along four properties: First, it speeds up during pleasant activities including pleasant (social) situations; second, it may decrease during unpleasant activities including unpleasant (social) situations; third, it may vanish during interesting activities and interesting social encounters; and last, it may be experienced as more pleasant with structured behavior. 
Concerning the three temporal dimensions of present, past, and future, we again found commonalities of experience between participants with ASD and a previous study on healthy individuals (Vogel et al., 2018a). Our material showed that the present had to be utilized and that it could be experienced or conceptualized both as extended in time or as a point in time.

However, several differences become apparent from our material. Whereas non-autistic individuals experienced the extension of the present as ranging from an activity (e.g. "I am presently reading.") to entire life phases (e.g. "I am presently retired") (Vogel et al., 2018a), participants with ASD seemed to restrict the present to a comparably fine-grained current activity. Furthermore, individuals with ASD described two different experiences of the present: either a snapshot-like momentary present or an extended duration of a meaningful activity.

This observed division of the present does not necessarily reflect incompatible experiential accounts because both demonstrate the interconnectedness between the three dimensions of time (Kupke, 2007; Kupke \& Vogeley, 2009; Vogel et al., 2018a). The extended present in this context is a present that is undisturbed by sudden events befalling it from the future. Our data demonstrate that the future was experienced as uncertain and scary, with fear being predominantly associated with the near future. Whereas non-autistic individuals experienced both fear and hope directed towards one uniform future (Vogel et al., 2018a), participants with ASD differentiated between a "general" or far away future on the one hand, and a near future associated with imminence, uncertainty, and fear on the other hand. We speculate that the irruption of the near future into the present breaks up the ongoing present activity and leaves behind an isolated, momentary impression associated with feelings of unreality and surprise. It seems likely that this observation of fear of the near future reflects the considerable incidence of co-occurring anxiety in ASD (Bellini, 2004).

This interconnected experience of present and future is further accentuated by the inclusion of the experience of the past. Although largely similar to the experience of the past as significant for the development of personality and character, as well as its inherent pastness and unchangeability in healthy control persons (Vogel et al., 2018a), the overly prevalent reference to memory reveals a division of time into isolated events. Again, we propose that this division further exemplifies the interconnectedness of the various aspects of time. The near future's uncertainty and imminence are compensated for and reduced through the implementation of behavioral structure and routine. By excessively planning future events, the future and the present become more predictable and more pleasant. This external sequencing of time carries over into the past which is memorized accordingly as a highly structured sequence of events.

Consequently, the experience of the present may reflect the performance of the constructed temporal sequence. If the present is experienced as a sequence of meaningful and pleasant activities, structure and routine prevailed. If the present is experienced predominantly as a sequence of individual instants, structure and routine have failed as a compensatory mechanism. In line with these considerations, it previously has been suggested that the ability to uphold routines and the period of time which may be planned successfully reflects the level of functioning in ASD (Boucher, 2001; Fein et al. 2013).

If this construction of a temporal sequence succeeds and a plan works out or routine remains undisturbed, it is recognized by a pleasant feeling or faded experience of the passage of time, and an otherwise mostly absent feeling of security. This consideration demonstrates the interconnectedness of structure and passage of time into an ongoing structured directedness (Kupke, 2007; Kupke \& Vogeley, 2009; Vogel et al., 2018a). Accordingly, it may be reasoned, that changes in the experience of the passage of time directly reflect changes of experience of the three dimensions of time (Vogel et al., 2018a; Vogel et al., 2018b). Vice versa, this observation explains the vulnerability of individuals with ASD to disturbances of their routines, a clinical feature commonly 
observed and described by the primary symptom of "restricted, repetitive patterns of behavior, interests, or activities" (American Psychiatric Association 2013, p.50).

Importantly, the structured directedness of time is not primarily disrupted. When focusing on the inner experience by means of introspection, or to a pleasant activity or situation, individuals with ASD appear to experience time comparable to individuals without ASD. The personally constructed routine remains intact and time passes regularly. In this case, the experience of time is undisturbed and may increase the frequency and intensity of flow experiences. Only when outside influences interrupt the self-imposed structure, the experience of time appears discontinued. We therefore propose the experience of time in ASD not to be dis-rupted or fragmented, as has been proposed for other psychiatric ailments (Wyllie, 2005; Fuchs, 2007; Gallagher, 2010; Allman \& Meck, 2012; Moore et al., 2013; Moskalewicz, 2015a; Northoff \& Stanghellini, 2016; Stanghellini et al., 2016; Yin et al., 2016; Fuchs \& van Duppen, 2017), but to be inter-rupted. A descriptive psychopathology of the syndrome of interrupted time experience is presented in Table 3.

\section{- Tbl. 3 app. here -}

Comparing these findings to the previous qualitative results from Zukauskas et al. (2009) we find that both are superimposable. Considering the "factual experience" (p.95), the present as "prolongation" of the past (p.104), the past as "fullest sense of time" (p.103-104), and abundant references to structure and routine, we can identify these constructs within our material as well. However, the authors did not primarily include the experience of the passage of time into their analysis. Therefore, they were unable to describe the phenomenon of time experience in ASD in its entirety. Also, the reported "prolongation of the past" into the present, would in our terms better be described as a prolongation of present and future into the past. It is not the past, which is prolonged into the present, but it is the structure within present and future which are reflected in the factual way the past is remembered.

\subsection{Implications for Research and Treatment}

Our observation of what we call interrupted experience of time in ASD leaves several implications. First and foremost the primary scope of our analysis of the inner time experience of participants with ASD was the data based identification of a comprehensive syndrome surrounding time experience in adults with ASD. An obvious follow-up study to this investigation could be the conceptualization and validation of a standardized questionnaire on time experience in ASD. A quantitative follow-up would also allow for complementary testing of variables potentially influencing the syndrome, such as e.g. gender and age.

Our qualitative findings cannot directly explain the putative underlying cognitive or neural mechanisms responsible for the examined variations in experience. The neural processes of Bayesian perceptual inference illustrated in the introduction may provide information and motivation for further neuroscientific investigation. We speculate that the comparatively slower integrative processes, correlating with (spatio-)temporal aspects of neural processes are disturbed in ASD and may be the cause of experiential disturbances (Hohwy et al., 2016; Vogel et al., 2018a), such as those reported herein. If according to the hypo prior hypothesis more time is needed to effectively come to a perceptual and experiential conclusion, and the surrounding circumstances do not provide the necessary time to do so, the underlying processes will literally be interrupted by the necessity to process the new and suddenly incoming sensory information. These interruptions will precipitate the experience of the passage of time as isolated, and momentary impressions one after the other. This will consequently lead to a conceptualization of time as comprised of isolated facts. 
The emerging difficulties will be more pronounced in situations with rapidly changing and nonforeseeable perceptual input, such as e.g. social interaction. The compensatory mechanism to reduce surprise and anxiety is the introduction of self-constructed priors in the form of routine and plans.

In reference to psychiatric diseases the brain's resting state has been implicated to be the most suitable candidate to explain disturbances of time experience. In this context it has been suggested, that the temporo-spatial structure of the brains resting state may correlate to the temporospatial structure of inner experience and thus of psychopathological symptoms (Northoff, 2016a; Northoff 2016b; Northoff \& Stanghellini, 2016). Further research on the spatio-temporal interrelationship through resting state studies may provide more detailed information on the implicit time experience not only in mental disease but also in ASD. As previous research has demonstrated differences within the Default Mode Network, i.e. the brains network primarily active during rest and idleness, between populations with ASD and non-autistic populations (e.g. Weng et al., 2010; Anderson et al. 2011; Joshi et al., 2017; Olivito et al. 2017), we may suppose that these features may very well be due to temporal disturbances.

Speculatively, an alteration in the Default Mode Network may be related to the results of this study, as it may be inferred from our data, that particularly idleness is not a desired state for an individual with ASD. It seems that a pleasant present is defined as an interesting and captivating activity. We may theoretically assume that the consequent effort to achieve an ongoing stream of pleasant activity and a reduction of future-directed anxiety, on a neuronal level may express a desired reduction in the activity of the Default Mode Network.

Apart from these diagnostic and research implications, an increased awareness of causes to suffering in ASD and a deeper knowledge of compensatory mechanisms as discussed herein may foster new therapeutic approaches, such as for instance instructions for structure implementation or training of general prospective planning capabilities.

\section{Limitations}

We conducted content analysis on 26 persons with ASD. We were able to identify a distinct experience of time in ASD. We infer from the analysis of our data that the explicit experience of time, open to conscious experience, is highly focused on implementing a stable structure and routine in order to decrease uncertainties lurking in the (near) future and to increase and stabilize present pleasantness. We surmise that depending on the level of cognitive function and the amount of external stressors the implemented structure succeeds or fails; in the case of failure causing distress and possibly descriptive autistic symptoms. We were able to explore and lay out in detail this syndrome of interrupted time experience in ASD. Our findings are heavily restricted to our specific sample of individuals with high functioning autism and exclusively describe inner experiences potentially affected by introspection impairments. The question of how this experience may be explained must be left open to speculation.

The method employed herein and the analysis of the collected material is limited to qualitative description and remains highly interpretative and vulnerable to oversimplification and false negatives. Despite their semi-quantitative report the results from this study constitute a qualitative account of time experience from adults with ASD. Frequency of responses does not illustrate a random sample measurement. Any comparison to results from other studies therefore must remain speculative, as statistical inference is not feasible. The postulated syndrome of interrupted time experience is not readily generalizable until it has been tested extensively. Accordingly we are unable to make any statement as to variance in symptom severity, presentation and occurrence. Although our analysis demonstrates and explains the features of interrupted time 
experience and provides a coherent and applicable concept of primary and explicit experience of time in ASD, the underlying (unconscious) cognitive processes and neural mechanisms will be the focus of future research. With our findings, we hope to inspire a novel research principal investigating the interrelationship of alterations in time experience both on an experiential and a neuronal level, as well as the integration of qualitative research and neuroscientific research in general.

\section{Compliance with Ethical Standards}

This study received no additional funding. Each author declares that he/she has no conflicts of interest. All procedures performed were in accordance with the ethical standards of the institutional and/or national research committee and with the 1964 Helsinki declaration and its later amendments or comparable ethical standards. Informed consent was obtained from all individual participants included in the study.

\section{References}

Allman, M. J. (2011). Deficits in temporal processing associated with autistic disorder. Frontiers in integrative neuroscience, 5(2).

Allman, M. J., \& DeLeon, I. G. (2009). "No Time Like the Present”: Time Perception in Autism (A. C. Giordano, Ed.)Causes and Risks for Autism. Nova Science Publishers, Inc.

Allman, M. J., DeLeon, I. G., \& Wearden, J. H. (2011). Psychophysical assessment of timing in individuals with autism. American journal on intellectual and developmental disabilities, 116(2), 165-178.

Allman M. \& Falter C. (2015). Abnormal timing and time perception in autism spectrum disorder? A review of the evidence. In Vatakis A., Allman M. (eds.), Time Distortions in Mind Temporal Processing in Clinical Populations (pp.37-56). Brill Academic Publishers, Leiden, The Netherlands.

Allman, M. J., \& Meck, W. H. (2012). Pathophysiological distortions in time perception and timed performance. Brain, 135(3), 656-677.

American Psychiatric Association (2013). Diagnostic and statistical manual of mental disorders (5th ed.). Arlington, VA: American Psychiatric Publishing.

Anderson, J. S., Nielsen, J. A., Froehlich, A. L., Dubray, M. B., Druzgal, T. J., Cariello, A. N., ... Lainhart, J. E. (2011). Functional connectivity magnetic resonance imaging classification of autism. Brain, 134, 3739-3751.

Baron-Cohen, S., Wheelwright, S., Skinner, R., Martin, J., \& Clubley, E. (2001). The autismspectrum quotient (AQ): Evidence from asperger syndrome/high-functioning autism, males and females, scientists and mathematicians. Journal of autism and developmental disorders, 31(1), 517.

Bebko, J. M., Weiss, J. A., Demark, J. L., \& Gomez, P. (2006). Discrimination of temporal synchrony in intermodal events by children with autism and children with developmental disabilities without autism. Journal of Child Psychology and Psychiatry, 47(1), 88-98.

Bellini, S. (2004). Social skill deficits and anxiety in high-functioning adolescents with autism spectrum disorders. Focus on Autism and Other Developmental Disabilities, 19(2), 78-86. 
Botbol, M., Cabon, P., Kermarrec, S., \& Tordjman, S. (2013). Biological and psychological rhythms: An integrative approach to rhythm disturbances in autistic disorder. Journal of Physiology Paris, 107(4), 298-309.

Boucher, J. (2001). Time-Parsing and Autism. In Hoerl C., McCormack T. (eds.) Time and memory: Issues in philosophy and psychology (pp.111-135). Oxford University Press.

Brock, J., Brown, C. C., Boucher, J., \& Rippon, G. (2002). The temporal binding deficit hypothesis of autism. Development and psychopathology, 14(2), 209-224.

Cassidy, S. A., Bradley, L., Bowen, E., Wigham, S., \& Rodgers, J. (2018). Measurement properties of tools used to assess depression in adults with and without autism spectrum conditions: A systematic review. Autism Research. In Press.

Csikszentmihályi, M. (1990). Flow: The Psychology of Optimal Experience. Harper \& Row.

Falter, C. M., \& Noreika, V. (2014). Time processing in developmental disorders: a comparative view. Subjective time: the philosophy, psychology, and neuroscience of temporality. MIT Press, Cambridge.

Falter, C. M., Noreika, V., Wearden, J. H., \& Bailey, A. J. (2012a). More consistent, yet less sensitive: interval timing in autism spectrum disorders. Quarterly Journal of Experimental Psychology, 65(11), 2093-2107.

Falter, C. M., Elliott, M. A., \& Bailey, A. J. (2012b). Enhanced visual temporal resolution in autism spectrum disorders. PLoS One, 7(3), e32774.

Falter, C. M., Braeutigam, S., Nathan, R., Carrington, S., \& Bailey, A. J. (2013). Enhanced access to early visual processing of perceptual simultaneity in autism spectrum disorders. Journal of autism and developmental disorders, 43(8), 1857-1866.

Fein, D., Barton, M., Eigsti, I. M., Kelley, E., Naigles, L., Schultz, R. T., ... Tyson, K. (2013). Optimal outcome in individuals with a history of autism. Journal of Child Psychology and Psychiatry and Allied Disciplines, 54(2), 195-205.

Foss-Feig, J. H., Kwakye, L. D., Cascio, C. J., Burnette, C. P., Kadivar, H., Stone, W. L., \& Wallace, M. T. (2010). An extended multisensory temporal binding window in autism spectrum disorders. Experimental Brain Research, 203(2), 381-389.

Friston, K. J., Stephan, K. E., Montague, R., \& Dolan, R. J. (2014). Computational psychiatry: the brain as a phantastic organ. The Lancet Psychiatry, 1(2), 148-158.

Fuchs, T. (2001). Melancholia as a desynchronization: Towards a psychopathology of interpersonal time. Psychopathology, 34, 179-186.

Fuchs, T. (2005). Implicit and Explicit Temporality. Philosophy, Psychiatry, \& Psychology, 12(3), $195-198$.

Fuchs, T. (2007). The temporal structure of intentionality and its disturbance in schizophrenia. Psychopathology, 40(4), 229-235.

Fuchs, T. (2013). Temporality and psychopathology. Phenomenology and the Cognitive Sciences, 12(1), 75-104. 
Fuchs, T., \& Van Duppen, Z. (2017). Time and Events: On the Phenomenology of Temporal Experience in Schizophrenia (Ancillary Article to EAWE Domain 2). Psychopathology, 50(1), $68-74$.

Gallagher, S. (2010). Consciousness of Time and the Time of Consciousness. Encyclopedia of Consciousness, 193-204.

Gepner, B., \& Féron, F. (2009). Autism: A world changing too fast for a mis-wired brain? Neuroscience and Biobehavioral Reviews, 33(8), 1227-1242.

Gil, S., Chambres, P., Hyvert, C., Fanget, M., \& Droit-volet, S. (2012). Children with Autism Spectrum Disorders Have "The Working Raw Material" for Time Perception. PloS One, 7(11), 1-10.

Glaser, B. \& Strauss, A. (2017). Discovery of grounded theory: Strategies for qualitative research. Routledge.

Hautzinger M., Bailer M., Worall, H., Keller, F. (1995). BDI: Beck-Depressionsinventar. Testhandbuch 2. überarbeitete Auflage. Bern: Verlag Hans Huber.

Hayes, A. F., \& Krippendorff, K. (2007). Answering the call for a standard reliability measure for coding data. Communication methods and measures, 1(1), 77-89.

Hohwy, J., Paton, B., \& Palmer, C. (2016). Distrusting the present. Phenomenology and the Cognitive Sciences, 15(3), 315-335.

Joshi, G., Arnold Anteraper, S., Patil, K., Semwal, M., Goldin, R., Furtak, S., ... WhitfieldGabrieli, S. (2017). Integration and Segregation of Default Mode Network Resting-state Functional Connectivity in Transition-age Males with High-functioning Autism Spectrum Disorder: A Proof of Concept Study. Brain Connectivity, 7(9), brain.2016.0483.

Krippendorff, K. (2004). Content analysis: An introduction to its methodology. Sage.

Kupke, C. (2009). Der Begriff Zeit in der Psychopathologie. Parodos Verlag.

Kupke, C. \& Vogeley, K. (2009). Constitution of Cognition in Time. In Baudson, T.G., Seemüller A., Dresler M. (Eds.) Chronobiology and Chronopsychology (pp. 121-149). Pabst Science Publishers, Lengerich, Germany.

Lepistö, T., Kujala, T., Vanhala, R., Alku, P., Huotilainen, M., \& Näätänen, R. (2005). The discrimination of and orienting to speech and non-speech sounds in children with autism. Brain research, 1066(1), 147-157.

Lepistö, T., Silokallio, S., Nieminen-von Wendt, T., Alku, P., Näätänen, R., \& Kujala, T. (2006). Auditory perception and attention as reflected by the brain event-related potentials in children with Asperger syndrome. Clinical Neurophysiology, 117(10), 2161-2171.

Mason, M. (2010). Sample Size and Saturation in PhD Studies Using Qualitative Interviews. Forum Qualitative Sozialforschung/Forum Qualitative Social Research, 11(3).

Mayring, P. (2014). Qualitative content analysis: theoretical foundation, basic procedures and software solution. Klagenfurt.

Mölder, B. (2016). Time in intersubjectivity: Some Tools for Analysis. In Mölder B., Arstilla, V., \& Øhrstrøm, P. (Eds.). Philosophy and Psychology of Time. Springer 2016. 
Moore, J. W., Cambridge, V. C., Morgan, H., Giorlando, F., Adapa, R., \& Fletcher, P. C. (2013). Time, action and psychosis: Using subjective time to investigate the effects of ketamine on sense of agency. Neuropsychologia, 51(2), 377-384.

Moskalewicz, M. (2015a). Disturbed temporalities. Insights from phenomenological psychiatry. Time \& Society, 1-19.

Moskalewicz, M. (2015b). Lived time disturbances of drug addiction therapy newcomers. A qualitative, field phenomenology case study at Monar-Markot Center in Poland. International Journal of Mental Health, 18(1), 3-20.

Nicholas, B., Rudrasingham, V., Nash, S., Kirov, G., Owen, M. J., \& Wimpory, D. C. (2007). Association of Per1 and Npas2 with autistic disorder: support for the clock genes/social timing hypothesis. Molecular psychiatry, 12(6), 581.

Nielsen, M. (2017). ADHD and Temporality: A Desynchronized Way of Being in the World. Medical Anthropology: Cross Cultural Studies in Health and Illness, 36(3), 260-272.

Noel, J. P., De Niear, M., Lazzara, N. S., \& Wallace, M. T. (2017). Uncoupling Between Multisensory Temporal Function and Non-Verbal Turn-Taking in Autism Spectrum Disorder. IEEE Transactions on Cognitive and Developmental Systems.

Noel, J. P., Lytle, M., Cascio, C., \& Wallace, M. T. (2018). Disrupted integration of exteroceptive and interoceptive signaling in autism spectrum disorder. Autism Research, 11(1), 194-205.

Northoff, G. (2016a). How do resting state changes in depression translate into psychopathological symptoms? From "Spatiotemporal correspondence" to "Spatiotemporal Psychopathology." Current Opinion in Psychiatry, 29(1), 18-24.

Northoff, G. (2016b). Spatiotemporal psychopathology I: No rest for the brain's resting state activity in depression? Spatiotemporal psychopathology of depressive symptoms. Journal of Affective Disorders, 190, 854-866.

Northoff, G., \& Stanghellini, G. (2016). How to Link Brain and Experience? Spatiotemporal Psychopathology of the Lived Body. Frontiers in Human Neuroscience, 10, 76.

Olivito, G., Clausi, S., Laghi, F., Tedesco, A. M., Baiocco, R., Mastropasqua, C., ... Leggio, M. (2017). Resting-State Functional Connectivity Changes Between Dentate Nucleus and Cortical Social Brain Regions in Autism Spectrum Disorders. Cerebellum, 16(2), 283-292.

Pellicano, E., \& Burr, D. (2012). When the world becomes 'too real': a Bayesian explanation of autistic perception. Trends in cognitive sciences, 16(10), 504-510.

Petzschner, F. H., Weber, L. A. E., Gard, T., \& Stephan, K. E. (2017). Computational Psychosomatics and Computational Psychiatry: Toward a Joint Framework for Differential Diagnosis. Biological Psychiatry. Elsevier Inc.

Pope, C., Ziebland, S., \& Mays, N. (2000). Qualitative research in health care: Analysing qualitative data. BMJ: British Medical Journal, 320, 114-116. 
Rippon, G., Brock, J., Brown, C., \& Boucher, J. (2007). Disordered connectivity in the autistic brain: challenges for the 'new psychophysiology'. International journal of psychophysiology, 63(2), 164-172.

Sandelowski, M. (1995). Sample Size in Qualitative Research. Research in Nursing \& Health, 18(2), 179-183.

Schmidt, K. H., \& Metzler, P. (1992). Wortschatztest: WST. Beltz.

Stanghellini, G., Ballerini, M., Presenza, S., Mancini, M., Raballo, A., Blasi, S., \& Cutting, J. (2016). Psychopathology of Lived Time: Abnormal Time Experience in Persons with Schizophrenia. Schizophrenia Bulletin, 42(1), 45-55.

Stanghellini, G., Ballerini, M., Presenza, S., Mancini, M., Northoff, G., \& Cutting, J. (2017). Abnormal Time Experiences in Major Depression: An Empirical Qualitative Study. Psychopathology, 50(2), 125-140.

Thönes, S., \& Oberfeld, D. (2015). Time perception in depression: A meta-analysis. Journal of Affective Disorders, 175, 359-372.

Tordjman, S., Davlantis, K. S., Georgieff, N., Geoffray, M. M., Speranza, M., Anderson, G. M., ... \& Vernay-Leconte, J. (2015). Autism as a disorder of biological and behavioral rhythms: toward new therapeutic perspectives. Frontiers in pediatrics, 3,1.

Trevarthen, C., \& Daniel, S. (2005). Disorganized rhythm and synchrony: Early signs of autism and Rett syndrome. Brain and Development, 27, S25-S34.

Varela, F. J. (1999). The Specious Present : A Neurophenomenology of Time Consciousness. In J. Petitot, F. J. Varela, B. Pachoud, \& J.-M. Roy (Eds.), Naturalizing Phenomenology: Issues in Contemporary Phenomenology and Cognitive Science (pp. 266-329). Stanford: Stanford University Press.

Vogel, D. H., Falter-Wagner, C. M., Schoofs, T., Krämer, K., Kupke, C., \& Vogeley, K. (2018a). Flow and structure of time experience-concept, empirical validation and implications for psychopathology. Phenomenology and the Cognitive Sciences, 1-24.

Vogel, D. H., Krämer, K., Schoofs, T., Kupke, C., \& Vogeley, K. (2018b). Disturbed Experience of Time in Depression-Evidence from Content Analysis. Frontiers in Human Neuroscience, 12, 66.

Vogeley, K., \& Kupke, C. (2007). Disturbances of time consciousness from a phenomenological and a neuroscientific perspective. Schizophrenia Bulletin, 33(1), 157-65.

Welsh, J. P., Ahn, E. S., \& Placantonakis, D. G. (2005). Is autism due to brain desynchronization?. International Journal of Developmental Neuroscience, 23(2), 253-263.

Weng, S. J., Wiggins, J. L., Peltier, S. J., Carrasco, M., Risi, S., Lord, C., \& Monk, C. S. (2010). Alterations of resting state functional connectivity in the default network in adolescents with autism spectrum disorders. Brain Res, 1313, 202-214. 
Wimpory, D., Nicholas, B., \& Nash, S. (2002). Social timing, clock genes and autism: a new hypothesis. Journal of Intellectual Disability Research, 46(4), 352-358.

World Health Organization (1992). The ICD-10 classification of mental and behavioral disorders: Clinical descriptions and diagnostic guidelines. Geneva: World Health Organization.

Woynaroski, T. G., Kwakye, L. D., Foss-Feig, J. H., Stevenson, R. A., Stone, W. L., \& Wallace, M. T. (2013). Multisensory speech perception in children with autism spectrum disorders. Journal of autism and developmental disorders, 43(12), 2891-2902.

Wyllie, M. (2005). Lived Time and Psychopathology. Philosophy, Psychiatry, \& Psychology, 12(3), 173-185.

Yin, B., Terhune, D. B., Smythies, J., \& Meck, W. H. (2016). Claustrum, consciousness, and time perception. Current Opinion in Behavioral Sciences, 8, 258-267.

Zukauskas, P. R., Silton, N., \& Assumpção Jr, F. B. (2009). Temporality and Asperger's syndrome. Journal of Phenomenological Psychology, 40(1), 85-106. 
Table 1: Demographics (Standard Deviation in Brackets)

\begin{tabular}{|c|c|}
\hline Gender & $m=18 ; f=8$ \\
\hline Mean Age & $41.6(+-9.8)$ \\
\hline $\begin{array}{l}\text { Mean Years of } \\
\text { Education }\end{array}$ & $18.7(+-4)$ \\
\hline Mean $A Q^{*}(n=17)$ & $42.0(+-3.7)$ \\
\hline Mean BDI ${ }^{\star \star}$ & $12.9(+-9)$ \\
\hline Mean Verbal IQ & $104.6(+-9)$ \\
\hline \multicolumn{2}{|c|}{$\begin{array}{l}\text { * Score above } 32 \text { indicates clinically } \\
\text { significant autistic traits. }\end{array}$} \\
\hline \multicolumn{2}{|c|}{$\begin{array}{l}\text { **Cut-Off Scores for BDI-II: } \\
\text { 0-13: minimal depression } \\
\text { 14-19: mild depression } \\
\text { 20-28: moderate depression } \\
\text { 29-63: severe depression. }\end{array}$} \\
\hline
\end{tabular}




\begin{tabular}{|c|c|c|}
\hline & Table 2 Categorical System and Examples from the Material & \\
\hline & The Passage of Time & \\
\hline K01 & During pleasant situations time passes quickly. & "Nice moments pass quickly." \\
\hline K02 & During captivating activities time passes quickly. & "With deep occupation time passes quickly." \\
\hline K03 & During captivating activities the passage of time is not felt. & "When I am occupied with special interests I do not feel the passage of time." \\
\hline K04 & During unpleasant activities time passes slowly. & "Activities that are no fun seem long." \\
\hline K05 & The passage of time is independent from the presence of others. & "In the presence of others nothing changes." \\
\hline K06 & The passage of time varies. & "The passage of time fluctuates." \\
\hline K07 & The sense of time is poor. & "I have hardly anv sense of time. I lack a sense of time during evervdav life." \\
\hline K08 & Timing devices are needed to tell time. & "I need a watch to sense time." \\
\hline K09 & With age time passes more rapidly. & "The years go by more quickly as you get older." \\
\hline K10 & In the presence of others time passes slowlv. & "With others time passes more slowlv." \\
\hline K11 & In the presence of others time passes quickly. & "In the presence of others time passes more quickly." \\
\hline K12 & In the presence of others the passage of time is not felt. & "When others are around I do not pay attention to time." \\
\hline K13 & Structure and routine influence the passage of time. & "With routine tasks time goes normal." \\
\hline K14 & Time passes quickly. & "Time passes too quickly." \\
\hline \multirow[t]{2}{*}{ K15 } & Time passes. & "The passage of time flows continuously without beginning or end." \\
\hline & The Present & \\
\hline K16 & The present has no duration/is not extended. & "The present is a point in time." \\
\hline K17 & The present has a duration/is extended. & "The present is an extended interval." \\
\hline K18 & The present is a current activity. & $\begin{array}{l}\text { "The present is the period of time during which I dedicate myself to doing one } \\
\text { thing." }\end{array}$ \\
\hline K19 & The present is the current moment. & "The present is experienced as momentary." \\
\hline K20 & The present is not real. & "The present is not graspable." \\
\hline K21 & The present is a sanctuary. & $\begin{array}{l}\text { "The present is experienced as positive because while in it I don't have to fear } \\
\text { anything." }\end{array}$ \\
\hline K22 & Time has no deeper meaning. & "Time is not important." \\
\hline \multirow[t]{2}{*}{ K23 } & The present has to be utilized. & "Every dav has to be lived. Your own life has to be mastered." \\
\hline & The Past & \\
\hline K24 & The past is memory. & "The past exists in memory." \\
\hline K25 & The past is variable. & "The past is constantly reevaluated." \\
\hline K26 & The past cannot be changed. & "The past is simply over and cannot be changed." \\
\hline K27 & The past shapes an individual. & "The past is a reservoir of experiences that can be used in the present." \\
\hline K28 & The past is experienced negative. & "The worst part of time is the past because of the harrowing memories." \\
\hline K29 & The past is experienced positive. & "The past stabalizes." \\
\hline \multirow[t]{2}{*}{ K30 } & The past is remembered well. & "The past is very vivid." \\
\hline & The Future & \\
\hline K31 & The future is uncertain. & "The future is unknown and uncertain." \\
\hline K32 & The future is being planned. & "I experience the future through planning. Safety through planning." \\
\hline K33 & The future is full of hope. & "The future is full of possibilities. It is malleable, so you have to influence it." \\
\hline K34 & The future scares. & "Because it is not foreseeable the future is connected to fear, worries, and \\
\hline K35 & The future consists of a near and a far future. & $\begin{array}{l}\text { "The future is the possibility that something happens on short notice or on long } \\
\text { notice." }\end{array}$ \\
\hline K36 & Rest. & $\begin{array}{l}\text { "Ticking Cuckoo clocks get on my nerves. Grandfather clocks with a gong are } \\
\text { better." }\end{array}$ \\
\hline
\end{tabular}


Table 3: Interrupted Time Experience

\begin{tabular}{|l|l|l|}
\hline Experience & Definition & $\begin{array}{l}\text { Relating Categories (\% of } \\
\text { participants) }\end{array}$ \\
\hline The faded out passage of time. & $\begin{array}{l}\text { The passage of time is hardly or } \\
\text { vaguely felt. A bad sense of time. } \\
\text { Fading is usually amplified by } \\
\text { structured and routine activity. }\end{array}$ & $\begin{array}{l}\text { K02 (35\%), K03 (23\%), K06 } \\
\text { K12\%), K07 (38\%), K08 (12\%), }\end{array}$ \\
\hline The present as confined activity. & $\begin{array}{l}\text { The present consists of one } \\
\text { distinct activity, usually deemed } \\
\text { important, necessary or } \\
\text { meaningful. Routine is } \\
\text { experienced as beneficial. } \\
\text { Interruptions are experienced as } \\
\text { distressing. }\end{array}$ & $\begin{array}{l}\text { K18 (35\%), K19 (42\%), K18 } \\
(35 \%), \mathrm{K} 21(15 \%), \mathrm{K} 23(38 \%)\end{array}$ \\
\hline The past as isolated memories. & $\begin{array}{l}\text { The past is described as a } \\
\text { repetition of facts. Although } \\
\text { meaningful and influential the past } \\
\text { is not necessarily part of the lived } \\
\text { present. }\end{array}$ & $\begin{array}{l}\text { K24 (31\%), K26 (19\%), K27 } \\
(46 \%), \mathrm{K} 30(23 \%)\end{array}$ \\
\hline The planned future. & $\begin{array}{l}\text { The future is experienced as } \\
\text { uncertain und frightening. The } \\
\text { future is rigorously planned to } \\
\text { avoid unexpected interruption. }\end{array}$ & $\begin{array}{l}\text { K31 (54\%), K32 (54\%), K34 } \\
(46 \%), \mathrm{K} 35 \text { (23\%) }\end{array}$ \\
\hline
\end{tabular}


Figure 1. The Time Questionnaire:

1. How do you experience the passage and flow of time?

2. Does the passage and flow of time change in the presence of other persons? If ,yes ', how?

3 . Does the passage and flow of time change in relation to surroundings or situation (e.g. workplace, spare time)? If yes, how?

4. Does the present appear to be a point in time or an extended period in time? If you experience the present as an extended period in time, how long does the present last?

5 . How do you experience the present and what does it mean to you?

6 . How do you experience the past and what does it mean to you?

7. How do you experience the future and what does it mean to you? 